\title{
Modelagem e simulação com o Arena para reduzir filas em um restaurante self service
}

Joseana Vinhote Melo

joseanavmelo@gmail.com

Universidade Federal do Amazonas

(UFAM), Manaus, Amazonas, Brasil

Juliana da Silva Ferreira

silva.julianaf93@gmail.com

Universidade Federal do Amazonas

(UFAM), Manaus, Amazonas, Brasil

Marcelo Augusto Oliveira da

Justa

marcelo.justa@gmail.com

Universidade do Estado do Amazonas (UEA), Manaus, Amazonas, Brasil

\author{
RESUMO
}

O objetivo deste estudo é utilizar a simulação e modelagem no Arena para verificar o comportamento dos processos do serviço self service oferecidos aos clientes pelo restaurante do Instituto de Ciências Humanas e Letras (ICHL) da Universidade Federal do Amazonas (UFAM), por meio das filas e também propor cenários alternativos, além do encontrado. Os cenários alternativos são modelos computacionais criados para reproduzir uma condição real ou não. No caso do cenário encontrado, foi modelado e simulado a situação do estabelecimento enquanto no ideal e utópico, apenas sugeriu-se possibilidades de melhorias através dos resultados obtido dos relatórios no Arena. Os relatórios mostraram que o cenário encontrado era uma oportunidade de melhoria, pois o processo self service era o gargalo do serviço. Com isso, criou-se um modelo computacional chamado de cenário ideal, onde o processo gargalo ganhou mais um processo e recurso e gerando melhores resultados em relação ao modelo anterior. Como ainda havia possibilidade para melhorar esse modelo, criou-se o utópico. O cenário utópico trouxe valores bem melhores do que o ideal, sendo este o último a ser criado, pois outro modelo após ele mudava o gargalo do self service para o processo caixa.
\end{abstract}

PALAVRAS-CHAVE: Self service. Arena. Modelagem. Simulação. 


\section{INTRODUÇÃO}

O restaurante do tipo self service é uma opção para pessoas que tem o dia a dia corrido e precisam economizar tempo, pois o serviço oferecido neste ambiente é de uma alimentação variada e rápida. Nesse modelo, no setor Norte da Universidade Federal do Amazonas (UFAM), pode-se verificar que há operações de três restaurantes. Dentre eles, está o restaurante do Instituto de Ciências Humanas e Letras (ICHL) que oferece aos clientes a possibilidade e a facilidade de chegarem e escolherem o que consumir.

Magnée (1996) afirma que o restaurante self service é aquele que já possui o preço da refeição estabelecida, o auto serviço se resume na apresentação de pratos em balcões onde o cliente escolhe o que irá consumir dentre as várias opções disponíveis. Desta forma, os restaurantes do tipo self service que utilizam o sistema por peso, no qual o cliente escolhe os alimentos e ao final paga pelo peso final contido em seu prato, são cada vez mais comuns em todo o país devido a fatores como praticidade, pratos variados, e custo acessível (MAGNÉE,1996; ABREU e TORRES, 2003; SANTOS et al., 2011).

Ocorre que ao chegar ao restaurante, o cliente se depara com filas. Desta forma, descaracterizando o local que antes deveria oferecer a facilidade do indivíduo chegar, servir-se, pagar, almoçar e ir embora para realizar seus afazeres de forma ágil, prática e simples. Portanto, filas que causam prejuízo ao empresário e incômodo para quem deseja usufruir o serviço.

De acordo com Fogliatti e Mattos (2007) um sistema de fila é qualquer processo aonde as pessoas chegam com o intuito de receber um serviço pelo qual esperam. Já o Ferreira (2009) diz que fila é a formação de uma fileira de pessoas que se colocam umas após as outras, em ordem cronológica de chegada a um ponto, ou também, uma estrutura de organização de dados na qual estes são recuperados na mesma sequência em que foram inseridos.

O surgimento da fila, em um sistema, dá-se pelo fato da demanda ser maior que a capacidade de prestação do serviço (GROSS et al., 2008). Sua presença causa experiências indesejáveis (GROSS et al., 2008; CARDOSO et al., 2010; ROMERO et al., 2010). E é por este motivo que existem ferramentas como Teoria das Filas que tem o intuito de auxiliar na redução das filas dos processos dos estabelecimentos.

A teoria das filas é um conjunto de conhecimentos matemáticos aplicados nas filas com o objetivo de prever possíveis acontecimentos e estabelecer um equilíbrio economicamente viável tanto para o cliente quanto o servidor (COSTA, 2006).

Uma aplicação simples e cotidiana da Teoria das Filas pode ser observada no trabalho desenvolvido por Scarp et al. (2013) que a utilizou para realização de análise no processo de atendimento nos caixas de um supermercado. Os autores coletaram os tempos dos intervalos entre as chegadas sucessivas de clientes nas filas e os tempos de atendimento dos caixas os quais, após simulação, provém padrões que auxiliam na tomada de decisão.

Outro estudo desenvolvido por Alves (2012) mostra que a Teoria das Filas foi utilizada juntamente a outra ferramenta, como a modelagem e simulação, para 
possibilitar a identificação de oportunidades de melhoria de um restaurante universitário do tipo self service.

Segundo o Longman Dictionary of Contemporary English (2003), a simulação é definida como uma atividade ou situação que reproduz uma condição real e que pode ser utilizada para testar qualquer coisa.

Harrell et al. (1996); Law e Kelton (1991) vão além e dizem que a simulação é o ato de imitar um procedimento real em menor tempo e com menor custo, permitindo um melhor estudo do que vai acontecer e de como consertar erros que gerariam grandes gastos.

Harrel e Tumay (1995) afirmam que a simulação tem se tornado uma ferramenta muito importante, poderosa e indispensável, pois permite a realização de análise de sistemas complexos além de ser utilizada por diversos profissionais das áreas de engenharia, administração, tecnologia entre outros.

Prado (2008) também complementa dizendo que, no mundo atual, a simulação tem inúmeras aplicações, tais como uma produção em uma manufatura, o funcionamento de um banco, um pedágio, um porto e até o movimento dos papéis em um escritório.

Nesse contexto, a simulação da pesquisa foi realizada por meio do software Arena, que é um dos mais conhecidos pelo mundo acadêmico e profissional. Aqui no Brasil, é representada pela empresa de Paragon Tecnologia Ltda. O software Arena foi criado em 1993, a partir da união de dois outros programas denominados SIMAN e CINEMA (PRADO, 1999).

Segundo Prado (2004) o SIMAN é uma linguagem de simulação. Em 1983, teve seu nome dado a um programa de computador pessoal (PC). Já o CINEMA, é um programa de animação de simulação para computadores, sendo este sido desenvolvido um ano após o SIMAN. Portanto, o Arena é um modelo computacional que possui diversas ferramentas e funcionalidades que sendo utilizadas de forma correta fornecem bases para uma análise criteriosa e eficiente capaz de viabilizar um maior entendimento do sistema estudado.

Segundo Law e Kelton (1991), o Arena é um programa que tem um ambiente gráfico integrado de simulação, que contém diversos recursos para modelagem, animação, análise estatística e de resultados. Na sua plataforma também há as seguintes ferramentas: analisador de dados de entrada (Input Analyzer), analisador de resultados (Output Analyzer) e analisador de processos (Process Analyzer).

Prado (2004), ainda diz que para construir uma representação do cenário que se deseja analisar é preciso que se faça inicialmente um desenho do objeto de estudo, onde este possibilite visualizar as estações de trabalho e as opções de fluxos entre as estações de trabalho para as entidades.

Souza (2009) ratifica que além de se identificar as entidades, as estações de trabalho e também as opções de fluxo, é preciso que também se faça a identificação das variáveis que irão dimensionar o sistema, pois a partir dos valores que serão assumidas por estas variáveis é que serão retiradas possíveis conclusões do que está sendo verificado.

Desta forma, a fim de utilizar as técnicas de simulação no Arena, realizou-se um estudo com coleta de dados tendo como base a formação de filas no 
restaurante self service do ICHL da UFAM. Portanto, o objetivo é verificar o comportamento dos processos e sugerir cenários que possam melhorar o serviço.

\section{DIAGNÓSTICO, MODELAGEM E SIMULAÇÃO}

O ambiente analisado para este estudo é o restaurante self service do ICHL que tem a demanda maior que sua oferta. Em uma hora de serviço, por exemplo, chegam ao restaurante 112 clientes e estes se dirigem primeiramente a mesa do buffet e somente depois ao caixa, porém só saem do caixa 25 pessoas das que entraram, isto é, os clientes estão passando mais tempo em fila.

A explicação para tal acontecimento é a formação de fila em torno da mesa de buffet, onde a média do tempo e a quantidade de cliente é de respectivamente 22,6 minutos e 43 pessoas. Já no caixa a media é de 01 pessoa atendida a cada 1 minuto aproximadamente. Estes valores foram quantificados por meio da modelagem e simulação no Arena e possibilitaram a criação de cenários alternativos para melhorar o serviço.

Para que houvesse a possibilidade de modelar, simular e criar cenários alternativos para o restaurante do ICHL foi necessário seguir inicialmente as seguintes etapas:

1) Visita inicial ao restaurante: Visitou-se inicialmente o restaurante com o intuito de entrevistar os proprietários, pedir autorização para realizar o estudo e entender o funcionamento do serviço oferecido, ou seja, saber quais eram as entradas, os processos e as saídas;

2) Construção de fluxograma: Construiu-se um fluxograma do serviço oferecido no restaurante aos clientes. A entrada do serviço foi considerada como a chegada dos clientes no restaurante no seu horário de funcionamento que é de 11:00 às 14:00 horas.

As operações de pegar bandeja, prato, talher, guardanapos e servir-se no buffet foram consideradas um único processo (processo de self service), tanto na coleta de dados como na simulação no Arena. As operações de pesar, pagar, pedir adicionais como água, suco e entre outros, que ocorre após o self service, também foram consideradas como um único processo (processo caixa). Considerou-se único porque a pessoa que pesa a refeição na balança eletrônica e dá o adicional ao cliente é mesma que recebe o pagamento.

Por fim, a saída foi definida como o deslocamento do cliente no caixa à mesa de refeição para consumir pelo que pagou. A Figura 1 abaixo mostra o fluxograma do sistema em questão.

Figura 1 - Fluxograma do serviço do restaurante

\section{Chegada}

\section{Self service}

\section{Caixa} Saída

Fonte: Autores (2016).

3) Definição de amostra: Após a construção do fluxograma, definiu-se que as amostras de dados coletados para o estudo seria o tempo entre as chegadas dos clientes, o tempo de self service e o tempo de atendimento no caixa. O tamanho da amostra da coleta de dados foi definido por uma especialista da área de 
modelagem e simulação, onde a mesma informou que 300 (trezentas) amostras de tempo de chegada, de self service e de atendimento no caixa seria suficiente para o estudo.

4) Coleta de dados: A coleta de dados ocorreu de forma aleatória, dias e semanas alternadas, dentre os horários de 11:00 e 14:00 horas e exigia no mínimo três pessoas com cronômetros para a retirada de tempos dos processos;

5) Bloco de notas: Nesta etapa, fez-se a inserção da coleta de dados tempo das 300 amostras de chegada, de self service e do caixa no bloco de notas para que estes ficassem no formato txt e pudessem ser utilizados pelo Input Analyzer;

6) Input Analyzer: Utilizou-se o Input Analyzer para realizar testes de aderência dos dados, isto é, para verificar a qualidade das amostras coletadas, onde estas poderiam ser aprovadas ou não. Todas as amostras foram aprovadas nos testes de aderência e seguem uma distribuição Weibull para os tempos de chegada, distribuição Normal para os tempos de self service e distribuição de Earlang para os tempos de caixa;

7) Modelagem e Simulação no Arena: Fez-se a modelagem e simulação no Arena do cenário encontrado, do ideal e utópico do restaurante. O objetivo desta etapa é modelar e simular o comportamento da realidade dos processos do serviço oferecidos aos clientes e com base nisso construir novos modelos, ideal e utópico, para melhorar o serviço se possível.

No Arena, modelou-se primeiramente o cenário encontrado (Figura 2), onde o bloco Create "Entrada" foi programado com a expressão 0.999 + WEIB(23.6, 0.666), em segundos. A entidade utilizada foi "Clientes", o número de entidades por chegada é 1 e o número máximo de chegadas foi definido como infinito. Em seguida, foi utilizado o bloco Process "SELF SERVICE". Em suas configurações foram utilizados dois recursos "R_LADO_A", "R_LADO_B" que fazem referência aos dois lados da mesa de self service e a expressão usada foi $\operatorname{NORM}(137,36)$, em segundos.

O próximo bloco utilizado foi o Leave "Leave_SELF". O tipo de conexão utilizada foi "Route". O tipo de estação escolhida foi Station e este bloco foi conectado ao Enter_CAIXA. O próximo bloco do processo é o Enter "Enter_CAIXA". Neste bloco, o tipo de estação escolhida foi Station. Em seguida, - bloco utilizado foi o Process "CAIXA". Neste foi utilizado um recurso "Atendente" e a expressão usada foi 11.5 + $\operatorname{ERLA}(8.57,3)$, em segundos. O último bloco utilizado foi o bloco Dispose "SAIDA". O mesmo representa o fim do processo estudado.

Figura 2 - Modelo do cenário encontrado

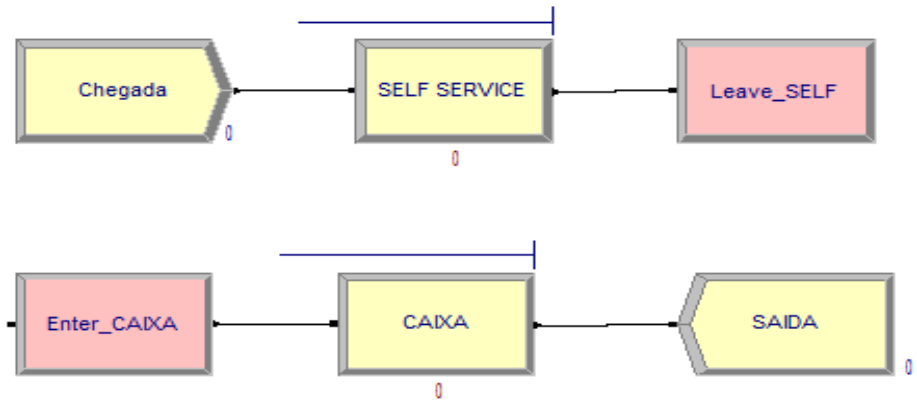

Fonte: Autores (2016). 
Após a construção do modelo no Arena, fez-se a simulação de 50 replicações em 1 hora de serviço com base nas informações programadas para obter dados para a análise. Esses são gerados por meio de relatórios no fim da simulação. No relatório, analisou-se o tempo, quantidade de cliente na fila e as taxas de utilização dos recursos como mesa do buffet e atendente nos processos de self service e caixa. Verificou-se também a quantidade total de pessoas que entravam e saíam do serviço.

O tempo mínimo, médio e máximo do cliente na fila do self service é de 0 , 22,6 e 50,4 minutos. No caso do caixa, é de $0,0,8$ e 1 minuto. A quantidade de clientes na fila do self service é no mínimo 0 , em média 43 e no máximo 116 enquanto no caixa é de no mínimo 0 , em média 1 e no máximo 1 . A média é 1 porque o resultado gerado no relatório do Arena deu um número decimal e equivale a uma pessoa na fila. Essa mesma lógica será aplicada nos restantes das médias nos próximos cenários do processo caixa. A taxa de utilização da mesa de buffet no self service é de $100 \%$ e do atendente no caixa é de $26,3 \%$. O número de clientes que chegam ao restaurante e entram na fila do self service é 116, porém dessa quantidade de pessoas apenas 25 saem do caixa e se dirigem a mesa para consumir seu alimento.

Com os valores encontrados, percebeu-se que a operação mais crítica era o self service, pois o tempo e o número de pessoas em fila eram alta demais o que ocasionava uma alta taxa de utilização da mesa do buffet e deixava o atendente ocioso no caixa. Também fazia a maior parte dos clientes ficarem mais tempo em fila do que sentado à mesa consumindo pelo que pagaram.

Sabendo-se que no self service havia oportunidade de melhoria, devido os valores não satisfatórios, criou-se outro modelo computacional no Arena, o cenário ideal. $O$ cenário ideal tem por objetivo melhorar o serviço no restaurante através da inclusão de mais uma mesa de buffet e consequentemente de mais um processo de self service. A Figura 3 abaixo mostra como ficou no Arena a nova configuração.

Figura 3 - Modelo do cenário ideal

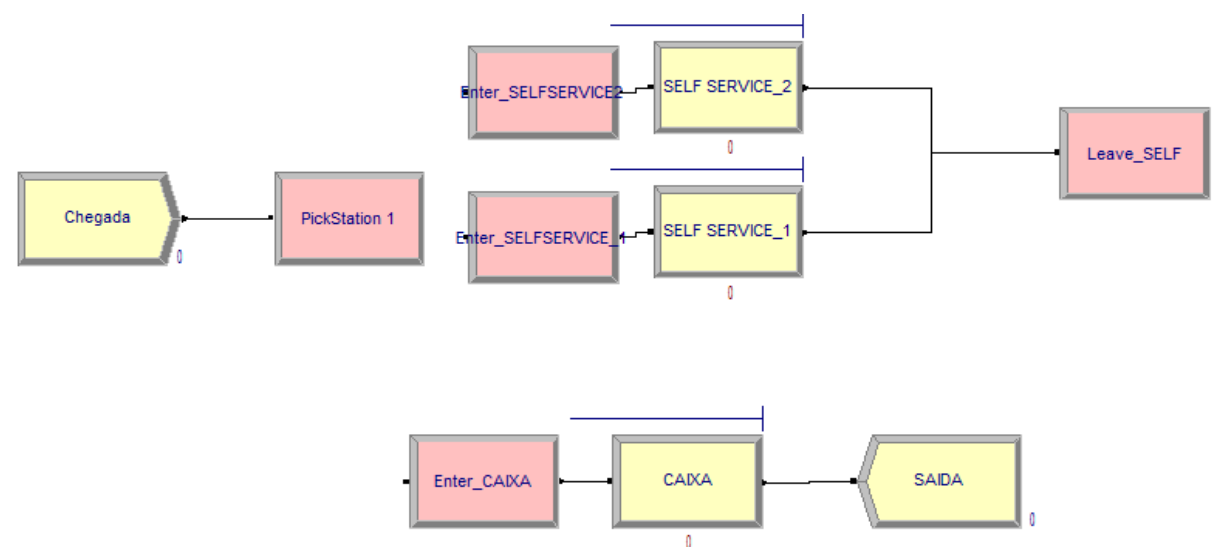

Fonte: Autores (2016)

No cenário ideal, as expressões dos blocos de representação do modelo simulado foram alteradas, pois houve acréscimo de mais um processo. Neste 
novo layout, incluíram-se novos blocos: PickStation 1, Enter_SELFSERVICE_1, Enter_SELFSERVICE2, Process (SELF SERVICE_2).

O resultado desse novo cenário foi bem mais satisfatório que o anterior, pois o tempo na fila do self service passou a ser de em média de 16,3 e no máximo 41,1 minutos. Já no caixa, o atendimento continuou em média 1 e de 1,8 no máximo. 0 número de clientes em fila nas mesas de buffet é no mínimo 0 , em média 15 e de no máximo 46 já no caixa esses valores são de 0,1 e 2 . A média 1 ainda continua devido o valor decimal equivalente a uma pessoa. A taxa de utilização do atendente no caixa aumentou para $52,5 \%$ e a das mesas diminui para $99,9 \%$ aproximadamente. A saída de clientes do serviço que vai desde a entrada no restaurante até a saída do caixa à mesa aumentou de 25 para 50 e isso significa que o serviço está mais rápido, principalmente o do self service.

Apesar de melhores resultados com o cenário ideal, percebeu-se ainda que havia possibilidade de melhora no tempo e no número pessoas na fila do self service, que o atendente poderia contribuir mais e a saída dos clientes do caixa à mesa também poderia aumentar.

Fez-se então a modelagem e simulação de um cenário utópico, que é um cenário melhor que o ideal. Este consiste no aumento do número de mais uma mesa de buffet e consequentemente de mais um processo self service em relação ao cenário anterior, o ideal. Neste novo layout utópico, foram acrescentados novos blocos: Enter_Self Service3 e um Process Self Service3, como mostra a Figura 4.

Figura 4 - Modelo do cenário utópico
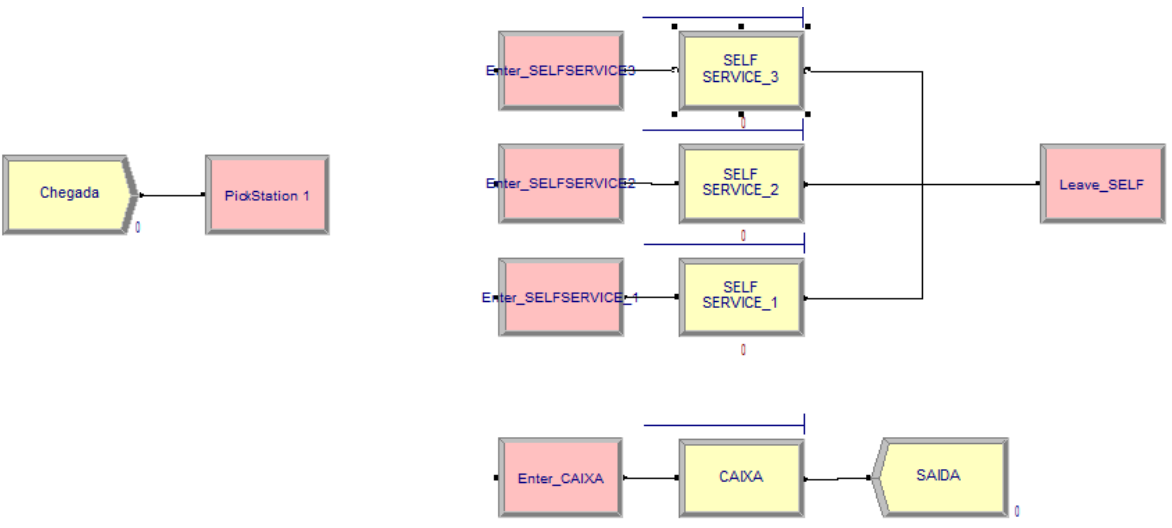

Fonte: Autores (2016).

Esse novo cenário também trouxe resultados positivos como a redução no tempo de fila do self service, onde o valor mínimo, médio e máximo é de respectivamente $0,10,4$ e 32,5 minutos. Já no caixa, o tempo médio continua 1 , porém o máximo é 3 , o que não traz prejuízo algum. A quantidade média de pessoas no self service diminui para 6 e no máximo 21, porém no caixa a média permanece 1 e o máximo é de 5 . No cenário anterior, tinha-se 50 clientes sentados à mesa consumindo suas refeições, com as mudanças esse valor aumentou para 75. A taxa de utilização da mesa de buffet diminui para 99,8\% e no caixa aumentou para $77,4 \%$

Após a construção desse cenário, fez-se outro modelo, porém, nele, constatou-se que o problema que antes era do processo de self service passaria a 
ser do caixa. Com isso, a modelagem e simulação de novos cenários pararam no utópico.

\section{RESULTADOS}

Os valores encontrados a partir da modelagem e simulação no Arena dos processos do restaurante do ICHL em 1 hora de serviço e com 50 replicações estão representados nos quadros seguir.

\section{Quadro 1 - Tempo dos clientes nos processos em minutos}

\begin{tabular}{|c|c|c|c|c|c|c|}
\hline \multicolumn{4}{|c|}{ Self service } & \multicolumn{3}{c|}{ Caixa } \\
\hline Cenário & Mínimo & Médio & Máximo & Mínimo & Médio & Máximo \\
\hline Encontrado & 0 & 22,6 & 50,4 & 0 & 0,8 & 1 \\
\hline Ideal & 0 & 16,3 & 41,1 & 0 & 1 & 1,8 \\
\hline Utópico & 0 & 10,4 & 32,5 & 0 & 1 & 3 \\
\hline
\end{tabular}

Fonte: Autores (2016).

O Quadro 1 representa o tempo que os clientes passam nas filas dos processos. No quadro, observa-se que os valores médio e máximo da operação no self service são maiores que no caixa, principalmente no cenário encontrado. Logo, isto significa que os clientes passam mais tempos no self service do que no caixa, pois o fluxo é lento e se ele é lento vai acarretar uma aglomeração de clientes em torno dele como mostra o Quadro 2.

Quadro 2 - Quantidade de cliente na fila dos processos

\begin{tabular}{|c|c|c|c|c|c|c|}
\hline \multicolumn{4}{|c|}{ Self service } & \multicolumn{3}{c|}{ Caixa } \\
\hline Cenário & Mínima & Média & Máxima & Mínima & Média & Máxima \\
\hline Encontrado & 0 & 43 & 116 & 0 & 1 & 1 \\
\hline Ideal & 0 & 15 & 46 & 0 & 1 & 2 \\
\hline Utópico & 0 & 6 & 21 & 0 & 1 & 5 \\
\hline
\end{tabular}

Fonte: Autores (2016).

No Quadro 2, observa-se que a quantidade média e máxima de pessoas são maiores no processo de self service e que o cenário encontrado é o que apresenta os maiores valores. Com isso, pode-se afirmar que o self service é o gargalo do restaurante e necessita de melhoria, pois tanto o Quadro 2 como o quadro 1 mostram dados que ratificam essa afirmação. É importante ressaltar que para fazer a melhoria é necessário levar em consideração os números do cenário encontrado.

No cenário encontrado, tem-se que os tempos médios e máximos são de 22,6 e 50,4 minutos e que a média e a máxima quantidade de clientes em fila é de respectivamente 43 e 116 no processo de self service e que para reduzir esses valores haveria a necessidade de inclusão de 1 processo de self service a mais no cenário ideal em relação ao encontrado. No utópico, a inclusão de 1 a mais do que o ideal. Lembrando que cada processo de self service vem com um recurso, ou seja, uma mesa de buffet. 
A melhoria realizada no cenário ideal e utópico reduziu tanto o tempo em fila como a quantidade de cliente no processo de self service, isto é, o cliente não vai enfrentar uma fila enorme e tão pouco ficar muito tempo nela. No caixa, os valores tempo e quantidade de clientes em fila não mudaram de forma significativa com a melhoria no self service, porém possibilitou uma melhor taxa de utilização do atendente.

A taxa de utilização atendente no caixa antes era baixa, $26,3 \%$, pois a clientela passava maior parte do tempo no self service, porém com a melhoria o colaborador passou a ficar menos ocioso na sua atividade e a atender mais clientes. A mesa do buffet manteve os valores de utilização bem altos, em torno de $100 \%$, mas por se tratar de um equipamento estático, sem partes móveis não houve sobrecarga, pois nesse caso não há problema nenhum em usar o máximo. O Quadro 3 representa as taxas de cada recurso dos processos.

Quadro 3 - Taxa de Utilização dos recursos nos processos

\begin{tabular}{|c|c|c|}
\hline Cenário & Mesa de Buffet (\%) & $\begin{array}{c}\text { Atendente } \\
(\%)\end{array}$ \\
\hline Encontrado & 100 & 26,3 \\
\hline Ideal & 99,9 & 52,5 \\
\hline Utópico & 99,8 & 77,4 \\
\hline
\end{tabular}

Fonte: Autores (2016)

Por fim, antes da melhoria, o número de clientes que chegavam ao restaurante e entravam na fila do self service era de 116, porém dessa quantidade de pessoas apenas 25 saíam do caixa e se dirigiam à mesa para consumir seu alimento. Com a melhoria esse valor de 25 subiu 50 no cenário ideal e no caso do utópico subiu para 75.

\section{CONSIDERAÇÕES FINAIS}

O restaurante do ICHL é um estabelecimento localizado no setor Norte da UFAM e que oferece aos seus clientes refeição por meio do serviço de self service. Neste local, o intuito era fazer um estudo com coleta de dados da formação de filas no restaurante, onde fosse possível verificar, por meio do Arena, o comportamento dos processos de self service, do caixa e sugerir cenários que pudessem melhorar o serviço.

O software Arena foi uma ferramenta imprescindível para a realização do estudo, pois foi por meio dele que houve a modelagem e simulação do cenário encontrado, ideal e utópico. O cenário encontrado era o retrato de como se encontrava os processos do serviço do restaurante, o cenário ideal e utópico são modelos computacionais criados a partir de um modelo já existente, o encontrado, onde o intuito deles é melhorar o serviço. Com a ajuda dos relatórios gerados pelo Arena, observou-se que a operação de self service era mais critica em relação a do caixa e necessitava de melhoria, pois o tempo e quantidade de clientes em fila apresentavam valores altos.

A melhoria no processo veio com a inclusão de mais uma operação de self service e mais um recurso, mesa de buffet. Essa inclusão foi feita tanto nos cenários ideal como no utópico. O cenário ideal tem dois processos de self service 
e um de caixa. Este mostrou, com a melhoria, que o tempo do cliente em fila diminuiu em cerca 6 em média e no máximo de 9 minutos e já no caixa houve pouca variação.

Ainda no cenário ideal, viu-se que o número médio e máximo de clientes na fila do self service reduziu para 28 e 70 . No caixa não teve tanta mudança, pois a média de clientes em fila permaneceu 1 e houve aumento de 1 pessoa a mais na quantidade máxima, porém já no caso da taxa de utilização do atendente houve um aumento em $26,2 \%$, ou seja, o colaborador passou ficar menos ocioso em sua atividade.

O cenário utópico tem três processos de self service e um de caixa. O tempo médio e máximo em fila, nesse caso, decresceu em aproximadamente 10 e 8 minutos. A quantidade média e máxima de clientes em fila teve um decréscimo 9 e 25 , o que significa menos pessoas no processo. Na operação do caixa a média de clientes continua a mesma, porém houve um acréscimo de 3 a mais na capacidade máxima, levando em consideração o modelo anterior, o que mostra que o colaborador pode atender mais pessoas e isso pode ser visto ainda pela taxa de utilização, onde houve um aumento de $24,9 \%$ a mais em relação aos 26 , $2 \%$.

A saída de clientes do serviço que vai desde a entrada no restaurante até a saída do caixa à mesa teve um aumento proporcional nos cenários ideal e utópico em relação encontrado que é de 25,50 e 75 . Isso mostra que o processo de self service está mais rápido e isto gera cliente satisfeito e mais lucro para o estabelecimento. Então, simularam-se apenas dois cenários, além do encontrado, para melhorar o serviço do restaurante do $\mathrm{ICHL}$ porque a criação de outro transferiria os problemas do processo de self service, o gargalo, para o caixa e isto não traria benefício nenhum.

Após a simulação do serviço do restaurante e a análise dos resultados, conclui-se que o objetivo principal do trabalho foi alcançado, conseguindo-se assim demonstrar o funcionamento do local bem próximo a sua realidade e sugerir alternativas mais eficientes por meio de outros modelos. Apesar das dificuldades encontradas durante o desenvolvimento da pesquisa como a coleta de dados, a construção dos cenários e da interpretação dos relatórios gerados pelo Arena obteve-se como resposta resultados satisfatórios.

Por fim, durante o desenvolvimento deste artigo, percebeu-se que o mesmo método de pesquisa, coleta e análise de dados usados neste estudo pode ser aplicado há outros tipos de serviços como a de um banco, por exemplo, onde pode ser realizado o estudo nas filas dos clientes no caixa eletrônico para sacar seu dinheiro, pagar contas na boca do caixa ou também quando necessitam de atendimentos mais específicos como retirada de cartão, negociação de contas, cancelamento de serviço entre outros.

Em todos esses casos que ocorrem no banco vai ter o tempo de chegada do cliente e o tempo de atendimento nos caixas, onde o modelo computacional do cenário encontrado no Arena vai ser bem simples: chegada dos clientes como entrada, atendimento dos clientes no caixa ou em outro setor como processo e a saída do setor de atendimento como a saída do sistema. Outra sugestão pode ser a inclusão de fila especial para idosos, gestantes, deficientes, crianças no colo na simulação. 


\title{
Modeling and simulation with Arena to reduce rows in a self service restaurant
}

\begin{abstract}
The objective of this study is to use the Arena simulation and modeling to verify the behavior of the self service processes offered to the clients by the restaurant of the Institute of Humanities and Letters (ICHL) of the Federal University of Amazonas (UFAM) and also to propose alternative scenarios, in addition to the one found. Alternative scenarios are computational models designed to reproduce a real condition or not. In the case of the scenario found, it was modeled and simulated the situation of the establishment while in the ideal and utopian, only suggested possibilities of improvements through the results obtained from the reports in the Arena. The reports showed that the scenario found was an opportunity for improvement because the self service process was the bottleneck of the service. Thus, a computational model was created called the ideal scenario, where the bottleneck process gained one more process and resource and generated better results than the previous model. As there was still possibility to improve this model, the Utopian was created. The utopian scenario brought much better values than the ideal, the latter to be created, since another model after it changed the bottleneck of self service to the cashier process.
\end{abstract}

KEYWORDS: Self service. Arena. Modeling. Simulation. 


\section{REFERÊNCIAS}

ABREU E. S.; TORRES E. A. F. S. Restaurante "por quilo": vale o quanto pesa? Uma avaliação do padrão alimentar em restaurantes em São Paulo, SP. Nutrire:

Revista da Sociedade Brasileira de Alimentação e Nutrição, v.25, p. 7-22, jun., 2003.

ALVES, R. Simulação da Dinâmica Operacional do Restaurante Universitário do Campus Medianeira da UTFPR: Um Estudo De Caso. 2012. 79 p. Monografia (Bacharel em Engenharia de Produção) - Universidade Tecnológica Federal do Paraná. 2012.

CARDOSO F. S.; FERNANDES JUNIOR, R. F.; SANTOS, Y. B. I. Pesquisa operacional: aplicação de Teoria de Filas no sistema de uma panificadora. In. ENCONTRO NACIONAL DE ENGENHARIA DE PRODUÇÃO 15, 2010, São Carlos. Anais... São Carlos: ABEPRO, 2010.

COSTA, L. C. Teorias das Filas: Apostila. Disciplina Teoria das Filas e Simulação, Curso de Ciência da Computação. Centro Tecnológico da Universidade Federal do Maranhão - UFMA. Maranhão, 2006.

FERREIRA, A. B. H. Novo Dicionário Aurélio. 4. ed. Curitiba: Positivo, 2009.

FOGLIATTI, M. C.; MATTOS, N. M. C. Teoria de Filas. Rio de Janeiro: Interciência, 2007.

GROSS, D.; SHORTLE, J. F.; THOMPSON, J. M.; HARRIS, C. M. Fundamentals of Queueing Theory. New Jersey: John Wiley \& Sons, 2008. crossref

HARREL, C.H.; TUMAY, K. Simulation made easy: a manager's guide. Engineering and Norcross; Management Press, 1995.

HARREL, C. R.; BATEMAN, R. E.; GOGG, T. J.; MOTT, J. R. A. System Improvement Using Simulation. Orem, Utah: PROMODEL Corporation. 1996.

LAW, A. M.; KELTON, W. D. Simulation Modelling and Analisys, 2.ed. New York: McGraw Hill, 1991.

Longman Dictionary of Contemporary English. 3. Ed., Pearson Education do Brasil LTDA, 2003.

MAGNÉE, Henri M. Manual do self service. São Paulo: Varela, 1996. 
PRADO, D. Usando o Arena em Simulação. Belo Horizonte: Desenvolvimento Gerencial, 1999.

PRADO, D. Usando o Arena em Simulação. 2. ed, Belo Horizonte: INDG Tecnologia e Serviços Ltda ,2004.

PRADO, D. Usando o ARENA em Simulação. 3. ed. Belo Horizonte: INDG Tecnologia e Serviços Ltda, 2008.

ROMERO, C. M.; SALES, D. S.; VILAÇA, L. L.; CHAVEZ, J. R. A.; CORTES, J. M. Aplicação da teoria das filas na maximização do fluxo de paletes em uma indústria química. Revista Eletrônica de Pesquisa Operacional para o Desenvolvimento, v. 2, n. 3, p. 226-231, set. / dez. 2010.

SANTOS, M. V.; PROENÇA, R. P.C; FIATES, G. M. R.; CALVO, M. C. M. Os Restaurantes por peso no contexto de alimentação saudável fora de casa. Revista de Nutrição, Campinas, v. 24, n. 4, ago. 2011.

SCARP, L. P. C.; TORRES, A. A. V. B.; SILVA, A. N. Simulação como ferramenta de tomada de decisão do processo de atendimento em empresa de varejo. Revista de Administração, Contabilidade e Economia, v. 12, n. 2, p. 395-430, jul./dez. 2013.

SOUZA, T. F. A simulação a eventos discretos como ferramenta de apoio à tomada de decisão em empresas do ramo de mineração: aplicação em uma unidade da Yamana. 2009. 16. P. Dissertação (Mestrado em Engenharia Mineral) - Programa de Pós-Graduação em Engenharia Mineral, Universidade Federal de Ouro Preto, Minas Gerais. 
Recebido: 29 jan. 2017

Aprovado: 22 set. 2017

DOI: 10.3895/gi.v13n2.5371

Como citar:

MELO, J. V.; FERREIRA, J. S.; JUSTA, M. A. O. Modelagem e simulação com o Arena para reduzir filas em um restaurante self service. R. Gest. Industr., Ponta Grossa, v. 13, n. 2, p. 146-159, jun./ago. 2017.

Disponível em: <https://periodicos.utfpr.edu.br/rgi>. Acesso em: XXX.

Correspondência:

Joseana Vinhote Melo

Rua dos Granitos, n 16, Tancredo Neves, Brasil.

Direito autoral: Este artigo está licenciado sob os termos da Licença Creative Commons-Atribuição 4.0

Internacional.

(c) (1) 